\title{
On a subclass of the generalized Janowski type functions of complex order
}

\author{
Öznur Özkan Kılıç*1 (1), Nuray Eroğlu² (1) \\ ${ }^{1}$ Department of Technology and Knowledge Management, Başkent University, Ankara, Turkey

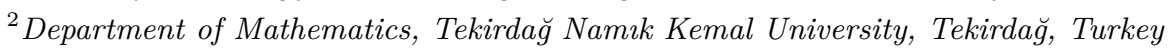

\begin{abstract}
In this paper, we introduce the class $\mathcal{J R}_{b}^{\lambda}(\alpha, \beta, \delta, A, B)$ of generalized Janowski type functions of complex order defined by using the Ruscheweyh derivative operator in the open unit disc $\mathbb{D}=\{z \in \mathbb{C}:|z|<1\}$. The bound for the $\mathrm{n}$-th coefficient and subordination relation are obtained for the functions belonging to this class. Some consequences of our main theorems are same as the results obtained in the earlier studies.
\end{abstract}

Mathematics Subject Classification (2010). 30C45

Keywords. analytic function, subordination, $\lambda$-spirallike function, $\lambda$-Robertson function, $\lambda$-close-to-spirallike function, $\lambda$-close-to-Robertson function, Ruscheweyh derivative operator

\section{Introduction and definitions}

Let $\mathcal{A}$ denote the class of functions of the form

$$
f(z)=z+\sum_{n=2}^{\infty} a_{n} z^{n}
$$

which are analytic in the open unit disc $\mathbb{D}=\{z \in \mathbb{C}:|z|<1\}$. Let $\mathcal{S}$ denote the subclass of $\mathcal{A}$ which are univalent in $\mathbb{D}$.

The hadamard product or convolution of two functions $f(z)=z+\sum_{n=2}^{\infty} a_{n} z^{n} \in \mathcal{A}$ and $g(z)=z+\sum_{n=2}^{\infty} b_{n} z^{n} \in \mathcal{A}$ denoted by $f * g$, is defined by

for $z \in \mathbb{D}$.

$$
(f * g)(z)=z+\sum_{n=2}^{\infty} a_{n} b_{n} z^{n}
$$

In 1975, Ruscheweyh [10] introduced a linear operator

with

$$
\mathcal{D}_{\mathcal{R}}^{\alpha} f(z)=\frac{z}{(1-z)^{\alpha+1}} * f(z)=z+\sum_{n=2}^{\infty} \varphi_{n}(\alpha) a_{n} z^{n}
$$

$$
\varphi_{n}(\alpha)=\frac{(\alpha+1)_{n-1}}{(n-1) !}
$$

*Corresponding Author.

Email addresses: oznur@baskent.edu.tr (Ö.Ö. Kılıç), neroglu@nku.edu.tr (N. Eroğlu)

Received: 16.08.2019; Accepted: 19.12.2019 
for $\alpha>-1$ and $(a)_{n}$ is Pochhammer symbol defined by

for $a \in \mathbb{C}$ and $\mathbb{N}=\{1,2,3, \ldots\}$.

$$
(a)_{n}=\frac{\Gamma(a+n)}{\Gamma(a)}
$$

Notice that

and

$$
\begin{gathered}
\mathcal{D}_{\mathcal{R}}^{0} f(z)=f(z), \\
\mathcal{D}_{\mathcal{R}}^{1} f(z)=z f^{\prime}(z)
\end{gathered}
$$

$$
\mathcal{D}_{\mathcal{R}}^{m} f(z)=\frac{z\left(z^{m-1} f(z)\right)^{m}}{m !}=z+\sum_{n=2}^{\infty} \frac{\Gamma(n+m)}{\Gamma(m+1)(n-1) !} a_{n} z^{n}
$$

for all $\alpha=m \in N_{0}=\{0,1,2, \ldots\}$.

In recent years, several authors obtained many interesting results for various subclasses of analytic functions defined by using the Ruscheweyh derivative operator.

Given two functions $f$ and $F$, which are analytic in the unit disk $\mathbb{D}$, we say that the function $f$ is subordinated to $F$, and write $f \prec F$ or $f(z) \prec F(z)$, if there exists a function $\omega$ analytic in $\mathbb{D}$ such that $|\omega(z)|<1$ and $\omega(0)=0$, with $f(z)=F(\omega(z))$ in $\mathbb{D}$.

In particular, if $F$ is univalent in $\mathbb{D}$, then $f(z) \prec F(z)$ if and only if $f(0)=F(0)$ and $f(\mathbb{D}) \subseteq F(\mathbb{D})$.

Let $\mathcal{P}$ denote the class of all functions of the form $p(z)=1+\sum_{n=1}^{\infty} p_{n} z^{n}$ that are analytic in $\mathbb{D}$ and for which $\Re p(z)>0$ in $\mathbb{D}$.

For arbitrary fixed numbers $A$ and $B$ with $-1 \leq B<A \leq 1$, Janowski [5] introduced the class $\mathcal{P}(A, B)$, defined by the subordination principle as follows:

$$
\mathcal{P}(A, B)=\left\{p: p(z) \prec \frac{1+A z}{1+B z}, \quad p(z)=1+p_{1} z+p_{2} z^{2}+\ldots\right\} .
$$

Also, if we take $A=1$ and $B=-1$, we obtain the well-known class $\mathcal{P}$ of functions with positive real part.

In 2006, Polatoglu [8] introduced the class $\mathcal{P}(A, B, \delta)$ of the generalization of Janowski functions as follows:

$$
\mathcal{P}(A, B, \delta)=\left\{p: p(z) \prec(1-\delta) \frac{1+A z}{1+B z}+\delta, \quad p(z)=1+p_{1} z+p_{2} z^{2}+\ldots\right\} .
$$

for arbitrary fixed numbers $A$ and $B$ with $-1 \leq B<A \leq 1,0 \leq \delta<1, z \in \mathbb{D}$.

Let $\mathcal{S}^{*}$ and $\mathcal{C}$ be the subclasses of $\mathcal{S}$ of all starlike functions and convex functions in $\mathbb{D}$, respectively. We also denote by $\mathcal{S}^{*}(\alpha)$ and $\mathfrak{C}(\alpha)$ the class of starlike functions of order $\alpha$ and the class of convex functions of order $\alpha$, where $0 \leq \alpha<1$, respectively.

In particular, we note that $\mathcal{S}^{*}:=\mathcal{S}^{*}(0)$ and $\mathcal{C}:=\mathcal{C}(0)$.

In [9], Reade introduced the class $\mathcal{S}^{*}$ of close-to-star functions as follows:

$$
\mathcal{C S}^{*}=\left\{f \in \mathcal{A}: \Re \frac{f(z)}{g(z)}>0 \text { and } g \in \mathcal{S}^{*}\right\}
$$

for all $z \in \mathbb{D}$. Also, we denote by $\operatorname{CS}^{*}(\beta)$ the class of close-to-star functions of order $\beta$ where $0 \leq \beta<1$. ( See Goodman [3]).

In [6], Kaplan introduced the class $\mathcal{C}$ of close-to-convex functions as follows:

$$
\mathcal{C}=\left\{f \in \mathcal{A}: \Re \frac{f^{\prime}(z)}{g^{\prime}(z)}>0 \text { and } g \in \mathcal{C}\right\}
$$


for all $z \in \mathbb{D}$. Also, we denote by $\mathcal{C}(\beta)$ the class of close-to-convex functions of order $\beta$ where $0 \leq \beta<1$. ( See Goodman [2]).

Clearly, we note that $\mathcal{C S}^{*}:=\mathcal{C S}^{*}(0)$ and $\mathcal{C} \mathcal{C}:=\mathcal{e} \mathcal{C}(0)$.

$f \in \mathcal{A}$ is an $\lambda$-spirallike function, $\mathcal{S P}^{\lambda}$, if and only if

$$
\Re\left[e^{i \lambda} \frac{z f^{\prime}(z)}{f(z)}\right]>0
$$

for some $|\lambda|<\frac{\pi}{2}, z \in \mathbb{D}$. The class of $\lambda$-spirallike functions was introduced by Špaček in [11].

Also, $f \in \mathcal{S P}^{\lambda}$ if and only if there exists a function $p \in \mathcal{P}$ such that

$$
f(z)=z \exp \left\{\cos \lambda e^{-i \lambda} \int_{0}^{z} \frac{p(t)-1}{t} d t\right\} .
$$

We note that the extremal function for the class of $\mathcal{S} \mathcal{P}^{\lambda}$

$$
f(z)=\frac{z}{(1-z)^{2 s}} \quad \text { where } \quad s=e^{-i \lambda} \cos \lambda,
$$

the $\lambda$-spiral koebe function.

$f \in \mathcal{A}$ is an $\lambda$-Robertson function, $\mathcal{R}^{\lambda}$, if and only if

$$
\Re\left[e^{i \lambda}\left(1+\frac{z f^{\prime \prime}(z)}{f^{\prime}(z)}\right)\right]>0
$$

for some $|\lambda|<\frac{\pi}{2}, z \in \mathbb{D}$.

Lemma 1.1. $f \in \mathcal{R}^{\lambda}$ if and only if there exists a function $p \in \mathcal{P}$ such that

$$
f^{\prime}(z)=\exp \left\{e^{-i \lambda} \int_{0}^{z} \frac{p(t) \cos \lambda-e^{i \lambda}}{t \cos \lambda} d t\right\}
$$

for some $|\lambda|<\frac{\pi}{2}, z \in \mathbb{D}$.

Proof. Suppose that $f \in \mathcal{R}^{\lambda}$. Since it is a $\lambda$-Robertson function, there exists a function $p \in \mathcal{P}$ such that

$$
e^{i \lambda}\left(1+\frac{z f^{\prime \prime}(z)}{f^{\prime}(z)}\right)=p(z) \cos \lambda \quad\left(|\lambda|<\frac{\pi}{2}, z \in \mathbb{D}\right) .
$$

From this equality, we can easily obtain (1.4).

Conversely, suppose that (1.4) holds. If we take the logarithmic derivative of (1.4), $f(z)$ belongs to $\mathcal{R}^{\lambda}$. So that, the proof is completed.

We note that $f \in \mathcal{R}^{\lambda}$ if and only if $z f^{\prime} \in \mathcal{S P}^{\lambda}$.

$f \in \mathcal{A}$ is an $\lambda$-close-to-spirallike function, $\mathcal{C S P}^{\lambda}$, if there exists a function $g \in \mathcal{S P}^{\lambda}$ such that

$$
\Re\left[\frac{f(z)}{g(z)}\right]>0
$$

for some $|\lambda|<\frac{\pi}{2}, z \in \mathbb{D}$.

We note that the extremal function for the class of $\operatorname{eSP}^{\lambda}$

$$
f(z)=\frac{z+z^{2}}{(1-z)^{2 s+1}}, \quad \text { where } \quad s=e^{-i \lambda} \cos \lambda,
$$

the $\lambda$-close-to-spiral koebe function. 
$f \in \mathcal{A}$ is an $\lambda$-close-to-Robertson function, $\mathcal{C} \mathcal{R}^{\lambda}$, if there exists a function $g \in \mathcal{R}^{\lambda}$ such that

$$
\Re\left[\frac{f^{\prime}(z)}{g^{\prime}(z)}\right]>0
$$

for some $|\lambda|<\frac{\pi}{2}, z \in \mathbb{D}$.

Haidan [4] introduced the class $\mathcal{S P}^{\lambda}(b)$ of $\lambda$-spirallike functions of complex order $b$ as follows:

$$
\mathcal{S P}^{\lambda}(b)=\left\{f \in \mathcal{A}: \Re\left\{1+\frac{e^{i \lambda}}{b \cos \lambda}\left(\frac{z f^{\prime}(z)}{f(z)}-1\right)\right\}>0\right\}
$$

for some $|\lambda|<\frac{\pi}{2}, b \in \mathbb{C}-\{0\}, z \in \mathbb{D}$.

Haidan [4] introduced the class $\mathcal{R}^{\lambda}(b)$ of $\lambda$-Robertson functions of complex order $b$ as follows:

$$
\mathcal{R}^{\lambda}(b)=\left\{f \in \mathcal{A}: \Re\left\{1+\frac{e^{i \lambda}}{b \cos \lambda}\left(\frac{z f^{\prime \prime}(z)}{f^{\prime}(z)}\right)\right\}>0\right\}
$$

for some $|\lambda|<\frac{\pi}{2}, b \in \mathbb{C}-\{0\}, z \in \mathbb{D}$.

Now, respectively, we introduce the classes of $\lambda$-close-to-spirallike functions of complex order $b$ and $\lambda$-close-to-Robertson functions of complex order $b$, denoted by $\operatorname{CSP}^{\lambda}(b)$ and $\mathrm{eR}^{\lambda}(b)$, as follows:

$$
\operatorname{CSP}^{\lambda}(b)=\left\{f \in \mathcal{A}: \Re\left\{1+\frac{1}{b}\left(\frac{f(z)}{g(z)}-1\right)\right\}>0, g \in \mathcal{S P}^{\lambda}\right\}
$$

and

$$
\operatorname{eR}^{\lambda}(b)=\left\{f \in \mathcal{A}: \Re\left\{1+\frac{1}{b}\left(\frac{f^{\prime}(z)}{g^{\prime}(z)}-1\right)\right\}>0, g \in \mathcal{R}^{\lambda}\right\}
$$

for some $|\lambda|<\frac{\pi}{2}, b \in \mathbb{C}-\{0\}, z \in \mathbb{D}$.

Definition 1.2. The class of generalized Janowski functions which are defined by Ruscheweyh derivative operator in $z \in \mathbb{D}$, denoted by $\mathcal{J} \mathcal{R}_{b}^{\lambda}(\alpha, \beta, \delta, A, B)$, is defined as

$\mathcal{J} \mathcal{R}_{b}^{\lambda}(\alpha, \beta, \delta, A, B)=\left\{f \in \mathcal{A}: 1+\frac{e^{i \lambda}}{b \cos \lambda}\left(\frac{\mathcal{D}_{\mathcal{R}}^{\alpha} f(z)}{\mathcal{D}_{\mathcal{R}}^{\beta} g(z)}-1\right) \prec(1-\delta) \frac{1+A z}{1+B z}+\delta, g \in \mathcal{S P}^{\lambda}\right\}$

for some $|\lambda|<\frac{\pi}{2}, b \in \mathbb{C}-\{0\}, \alpha>-1, \beta>-1,0 \leq \delta<1,-1 \leq B<A \leq 1, z \in \mathbb{D}$.

Nothing that the class $\mathcal{J} \mathcal{R}_{b}^{\lambda}(\alpha, \beta, \delta, A, B)$ include several subclasses which have important role in the analytic and geometric function theory.

By specializing the parameters $\alpha, \beta, \delta, \lambda, b$ and $A, B$, we obtain the following subclasses studied earlier:

(1) $\mathcal{C S}_{b}^{*}(\delta, A, B):=\mathcal{J P}_{b}^{0}(0,0, \delta, A, B)$ is the class of the generalized Janowski type close-to-star functions of complex order $b$,

(2) $\mathcal{C S}_{b}^{*}(A, B):=\mathcal{J} \mathcal{R}_{b}^{0}(0,0,0, A, B)$ is the class of the Janowski type close-to-star functions of complex order $b$,

(3) $\mathcal{C S}^{*}(A, B):=\mathcal{J R}_{1}^{0}(0,0,0, A, B)$ is the class of the Janowski type close-to-star functions,

(4) $\mathcal{C S}^{*}(\eta):=\mathcal{J R}_{1}^{0}(0,0,0,1-2 \eta,-1)$ is the class of the close-to-star functions of order $\eta$

(5) $\mathcal{C S}^{*}:=\mathcal{J} \mathcal{R}_{1}^{0}(0,0,0,1,-1)$ is the class of the close-to-star functions,

(6) $\mathcal{C C}_{b}(\delta, A, B):=\mathcal{J R}_{b}^{0}(1,0, \delta, A, B)$ is the class of the generalized Janowski type close-to-convex functions of complex order $b$,

(7) $\mathcal{C}_{b}(A, B):=\mathcal{J} \mathcal{R}_{b}^{0}(1,0,0, A, B)$ is the class of the Janowski type close-to-convex functions of complex order $b$, 
(8) $\mathcal{C}(A, B):=\mathcal{J P}_{1}^{0}(1,0,0, A, B)$ is the class of the Janowski type close-to-convex functions,

(9) $\mathcal{C}(\eta):=\mathcal{J} \mathcal{R}_{1}^{0}(1,0,0,1-2 \eta,-1)$ is the class of the close-to-convex functions of order $\eta$,

(10) $\mathcal{C} \mathfrak{C}:=\mathcal{J} \mathcal{R}_{1}^{0}(1,0,0,1,-1)$ is the class of the close-to-convex functions.

Lemma 1.3. [1] If the function $p(z)$ of the form

$$
p(z)=1+\sum_{n=1}^{\infty} p_{n} z^{n}
$$

is analytic in $\mathbb{D}$ and

$$
p(z) \prec \frac{1+A z}{1+B z},
$$

then $\left|p_{n}\right| \leq A-B$, for $n \in \mathbb{N},-1 \leq B<A \leq 1$.

Theorem 1.4. [3] If $f \in \mathcal{S P}^{\lambda}$, then

$$
\left|a_{n}\right| \leq \prod_{k=1}^{n-1} \frac{|k+2 s-1|}{k},
$$

where $s=e^{-i \lambda} \cos \lambda,|\lambda|<\frac{\pi}{2}, z \in \mathbb{D}$.

\section{Subordination result and their consequences}

Theorem 2.1. $f(z) \in \mathcal{J P}_{b}^{\lambda}(\alpha, \beta, \delta, A, B)$ if and only if

$$
\frac{\mathcal{D}_{\mathcal{R}}^{\alpha} f(z)}{\mathcal{D}_{\mathcal{R}}^{\beta} g(z)}-1 \prec \frac{(1-\delta)(A-B) b e^{-i \lambda} \cos \lambda z}{1+B z} .
$$

Proof. Suppose that $f \in \mathcal{J R}_{b}^{\lambda}(\alpha, \beta, \delta, A, B)$. Using the subordination principle, we write

$$
1+\frac{e^{i \lambda}}{b \cos \lambda}\left(\frac{\mathcal{D}_{\mathcal{R}}^{\alpha} f(z)}{\mathcal{D}_{\mathcal{R}}^{\beta} g(z)}-1\right)=(1-\delta) \frac{1+A \omega(z)}{1+B \omega(z)}+\delta .
$$

After simple calculations, we get

$$
\frac{e^{i \lambda}}{b \cos \lambda}\left(\frac{\mathcal{D}_{\mathcal{R}}^{\alpha} f(z)}{\mathcal{D}_{\mathcal{R}}^{\beta} g(z)}-1\right)=\frac{(1-\delta)(A-B) \omega(z)}{1+B \omega(z)} .
$$

Thus, this equality is equivalent to (2.1). Similarly, the other side is proved.

In Theorem 2.1, if we choice special values for $\alpha, \beta, \delta, \lambda, b$ and $A, B$ we get the following corollaries.

Corollary 2.2. $f(z) \in \operatorname{eSP}^{\lambda}(b)$ if and only if

$$
\frac{f(z)}{g(z)}-1 \prec \frac{2 b e^{-i \lambda} \cos \lambda z}{1-z}
$$

and this result is as sharp as the function

$$
\frac{2 b e^{-i \lambda} \cos \lambda z}{(1-z)^{2 s+1}}, \quad \text { where } s=e^{-i \lambda} \cos \lambda .
$$

Proof. We let $\alpha=\beta=\delta=0$ and $A=1, B=-1$ in Theorem 2.1. 
Corollary 2.3. $f(z) \in \mathcal{C S}^{*}(A, B)$ if and only if

and this result is as sharp as the function

$$
\frac{f(z)}{g(z)}-1 \prec \frac{(A-B) z}{1+B z}
$$

$$
\frac{1+A z}{1+B z} \cdot \frac{z}{(1-z)^{2}}
$$

Proof. We let $\lambda=\alpha=\beta=\delta=0$ and $b=1$ in Theorem 2.1.

Corollary 2.4. $f(z) \in \mathcal{C S}^{*}$ if and only if

$$
\frac{f(z)}{g(z)}-1 \prec \frac{2 z}{1-z}
$$

and this result is as sharp as the function

$$
\frac{1+z}{1-z}
$$

Proof. We let $\lambda=\alpha=\beta=\delta=0$ and $b=1, A=1, B=-1$ in Theorem 2.1.

Corollary 2.5. $f(z) \in \mathcal{R}^{\lambda}(b)$ if and only if

$$
\frac{z f^{\prime}(z)}{g(z)}-1 \prec \frac{2 b e^{-i \lambda} \cos \lambda z}{1-z} .
$$

Proof. We let $\alpha=1, \beta=\delta=0$ and $A=1, B=-1$ in Theorem 2.1.

Corollary 2.6. $f(z) \in \mathcal{C} \mathcal{C}(A, B)$ if and only if

$$
\frac{z f^{\prime}(z)}{g(z)}-1 \prec \frac{(A-B) z}{1+B z} .
$$

Proof. We let $\lambda=\beta=\delta=0$ and $\alpha=1, b=1$ in Theorem 2.1.

Corollary 2.7. $f(z) \in \mathcal{C} \mathcal{C}$ if and only if

$$
\frac{z f^{\prime}(z)}{g(z)}-1 \prec \frac{2 z}{1-z}
$$

and this result is as sharp as the function

$$
\frac{1+z}{1-z}
$$

Proof. We let $\lambda=\beta=\delta=0$ and $\alpha=1, b=1, A=1, B=-1$ in Theorem 2.1.

\section{Coefficient estimates and their consequences}

Lemma 3.1. If the function $\phi(z)$ of the form

$$
\phi(z)=1+\sum_{n=1}^{\infty} \phi_{n} z^{n}
$$

is analytic in $\mathbb{D}$ and

$$
\phi(z) \prec(1-\delta) \frac{1+A z}{1+B z}+\delta,
$$

then

$$
\left|\phi_{n}\right| \leq(A-B)(1-\delta)
$$

for $0 \leq \delta<1,-1 \leq B<A \leq 1, n \in \mathbb{N}, z \in \mathbb{D}$. 
Proof. Suppose that $\phi(z) \prec(1-\delta) \frac{1+A z}{1+B z}+\delta$ for $\phi(z)=1+\sum_{n=1}^{\infty} \phi_{n} z^{n}$. Using the subordination principle, we write

$$
\phi(z)=(1-\delta) \frac{1+A \omega(z)}{1+B \omega(z)}+\delta .
$$

From (3.2), we get

$$
\kappa(z)=\frac{\phi(z)-\delta}{(1-\delta)}=\frac{1+A \omega(z)}{1+B \omega(z)} .
$$

By using Lemma 1.3 for the above function $\kappa(z)$, we get

$$
\left|\frac{\phi_{n}}{1-\delta}\right| \leq A-B
$$

This inequality is equivalent to (3.1).

Theorem 3.2. If the function $f(z) \in \mathcal{A}$ be in the class $\mathcal{J \mathcal { R }}_{b}^{\lambda}(\alpha, \beta, \delta, A, B)$, then

$$
\begin{aligned}
\left|a_{n}\right| \leq & \frac{1}{|b| \varphi_{n}(\alpha)} \\
& \times\left(|b| \varphi_{n}(\beta) \prod_{k=1}^{n-1} \frac{|k+2 s-1|}{k}+(A-B)(1-\delta)\left[\sum_{m=1}^{n-1} \varphi_{n-m}(\beta) \prod_{k=1}^{n-(m+1)} \frac{|k+2 s-1|}{k}\right]\right),
\end{aligned}
$$

where $s=e^{-i \lambda} \cos \lambda,|\lambda|<\frac{\pi}{2}, b \in \mathbb{C}-\{0\}, \alpha>-1, \beta>-1,0 \leq \delta<1,-1 \leq B<A \leq 1$, $z \in \mathbb{D}$.

Proof. Since $f \in \mathcal{J R}_{b}^{\lambda}(\alpha, \beta, \delta, A, B)$, there are analytic functions $g, \phi: \mathbb{D} \longmapsto \mathbb{D}$ such that $g(z)=z+\sum_{n=2}^{\infty} b_{n} z^{n} \in \mathcal{S P}^{\lambda}, \phi(z)=1+\sum_{n=1}^{\infty} \phi_{n} z^{n}$ and $\omega(z)$ is a Schwarz function as in Lemma 3.1 such that

$$
1+\frac{e^{i \lambda}}{b \cos \lambda}\left(\frac{\mathcal{D}_{\mathcal{R}}^{\alpha} f(z)}{\mathcal{D}_{\mathcal{R}}^{\beta} g(z)}-1\right)=(1-\delta) \frac{1+A \omega(z)}{1+B \omega(z)}+\delta=\phi(z)
$$

for $z \in \mathbb{D}$. Then (3.4) can be written as

$$
\mathcal{D}_{\mathcal{R}}^{\alpha} f(z)=\{1+s b[\phi(z)-1]\} \mathcal{D}_{\mathcal{R}}^{\beta} g(z)
$$

or

$$
z+\sum_{n=2}^{\infty} \varphi_{n}(\alpha) a_{n} z^{n}=z+\sum_{n=2}^{\infty}\left\{\varphi_{n}(\beta) b_{n}+s b \sum_{m=1}^{n-1} \varphi_{n-m}(\beta) b_{n-m} \phi_{m}\right\} z^{n} .
$$

Equating the coefficients of like powers of $z$, we get

$$
\begin{gathered}
\varphi_{2}(\alpha) a_{2}=\varphi_{2}(\beta) b_{2}+s b \phi_{1}, \\
\varphi_{3}(\alpha) a_{3}=\varphi_{3}(\beta) b_{3}+s b\left[\varphi_{2}(\beta) b_{2} \phi_{1}+\phi_{2}\right]
\end{gathered}
$$

and

$$
\varphi_{n}(\alpha) a_{n}=\varphi_{n}(\beta) b_{n}+s b\left[\varphi_{n-1}(\beta) b_{n-1} \phi_{1}+\varphi_{n-2}(\beta) b_{n-2} \phi_{2}+\ldots+\phi_{n-1}\right] .
$$

By using Lemma 3.1 and Theorem 1.4, we get (3.3).

Corollary 3.3. Let $f(z) \in \mathcal{A}$ be in the class $\operatorname{CSP}^{\lambda}(b)$, then

$$
\left|a_{n}\right| \leq \frac{1}{|b|}\left(|b| \prod_{k=1}^{n-1} \frac{|k+2 s-1|}{k}+2\left[\sum_{m=1}^{n-1} \prod_{k=1}^{n-(m+1)} \frac{|k+2 s-1|}{k}\right]\right),
$$

where $s=e^{-i \lambda} \cos \lambda,|\lambda|<\frac{\pi}{2}, b \in \mathbb{C}-\{0\}, z \in \mathbb{D}$.

Proof. In Theorem 3.2, we take $\alpha=\beta=\delta=0$ and $A=1, B=-1$. 
Corollary 3.4. [7] Let $f(z) \in \mathcal{A}$ be in the class $\mathcal{C S}^{*}(A, B)$, then

$$
\left|a_{n}\right| \leq n+\frac{(A-B)(n-1) n}{2},
$$

where $-1 \leq B<A \leq 1, z \in \mathbb{D}$.

Proof. In Theorem 3.2, we take $\alpha=\beta=\delta=\lambda=0$ and $b=1$.

Corollary 3.5. [7] Let $f(z) \in \mathcal{A}$ be in the class SS*, then $^{*}$

$$
\left|a_{n}\right| \leq n^{2}
$$

where $z \in \mathbb{D}$.

Proof. In Theorem 3.2, we take $\alpha=\beta=\delta=\lambda=0$ and $b=1$.

Corollary 3.6. Let $f(z) \in \mathcal{A}$ be in the class $\mathcal{R}^{\lambda}(b)$, then

$$
\left|a_{n}\right| \leq \frac{1}{|b| n}\left(|b| \prod_{k=1}^{n-1} \frac{|k+2 s-1|}{k}+2 \sum_{m=1}^{n-1} \prod_{k=1}^{n-(m+1)} \frac{|k+2 s-1|}{k}\right),
$$

where $s=e^{-i \lambda} \cos \lambda,|\lambda|<\frac{\pi}{2}, b \in \mathbb{C}-\{0\}, z \in \mathbb{D}$.

Proof. In Theorem 3.2, we take $\alpha=1, \beta=\delta=0$ and $A=1, B=-1$.

Corollary 3.7. [7] Let $f(z) \in \mathcal{A}$ be in the class $\mathcal{C C}(A, B)$, then

$$
\left|a_{n}\right| \leq 1+\frac{(A-B)(n-1)}{2}
$$

where $-1 \leq B<A \leq 1, z \in \mathbb{D}$.

Proof. In Theorem 3.2, we take $\alpha=1, \beta=\delta=\lambda=0$ and $b=1$.

Corollary 3.8. [7] Let $f(z) \in \mathcal{A}$ be in the class $\mathcal{C}$, then

$$
\left|a_{n}\right| \leq n
$$

where $z \in \mathbb{D}$.

Proof. In Theorem 3.2, we take $\alpha=1, \beta=\delta=\lambda=0$ and $A=1, B=-1, b=1$.

\section{References}

[1] R.M. Goel and B.C. Mehrok, A subclass of univalent functions, Houston J. Math. 8, 343-357, 1982.

[2] A.W. Goodman, On close-to-convex functions of higher order, Ann. Univ. Sci. Budapest Eötvös Sect. Math. 15, 17-30, 1972.

[3] A.W. Goodman, Univalent Functions, Vol II. Somerset, NJ, USA Mariner, 1983.

[4] M.M. Haidan and F.M. Al-Oboudi, Spirallike functions of complex order, J. Natural Geom. 19, 53-72, 2000.

[5] W. Janowski, Some extremal problems for certain families of analytic functions, Ann. Polon. Math. 28, 297-326, 1973.

[6] W. Kaplan, Close-to-convex schlicht functions, Michigan Math. J. 1, 169-185, 1952.

[7] Ö.Ö. Kılıç, Coefficient Inequalities for Janowski type close-to-convex functions associated with Ruscheweyh Derivative Operator, Sakarya Uni. J. Sci. 23 (5), 714-717, 2019.

[8] Y. Polatoğlu, M. Bolcal, A. Şen and E. Yavuz, A study on the generalization of Janowski functions in the unit disc, Acta Math. Aca. Paed. 22, 27-31, 2006.

[9] M.O. Reade, On close-to-convex univalent functions, Michigan Math. J. 3, 59-62, 1955. 
[10] S. Ruscheweyh, New criteria for univalent functions, Proc. Amer. Math Soc. 49 (1), 109-115, 1975.

[11] L. Špaček, Prispevek $k$ teorii funcki prostych, Casopis Pest. Mat. a Fys. 62, 12-19, 1932. 